Now the Royal National Institute for the Blind in London prints 100000 books, 395000 newspapers, and 130000 magazines a year in Braille. Skill in reading it comes more easily to the young than the elderly, but there is no reason why blind patients of any age should not be encouraged to use Braille. Sensitivity in the tips of the fingers and an ability to concentrate are essential. The Moon system, developed after the Braille system and also consisting of raised marks, does not require so much sensitivity but is not so widely used and the libraries are not so comprehensive.

A "blind" person is not necessarily a person without sight. To be registered as blind a person must have insufficient sight to do work for which sight is essential. Many persons registered as blind can walk about by themselves and, with care, can cross busy roads. Some of them if they do not wish to study Braille are helped to do some reading by the use of visual aids. These take various forms, but the simplest is the ordinary magnifying glass; others are compound lenses, similar to opera glasses, in various strengths and combinations. Macular degeneration, common in later life, is a typical example of an ocular disease which causes difficulty in reading but whose effects can be mitigated by the use of a low-vision aid. Reading is not so easy as with normal eyes, because lowvision aids do not have a big field of vision. The reader has to some extent to read along a line word by word in contrast to the ordinary reading, when the meaning is taken in line by line. Reading with a low-vision aid tends, therefore, to be slow. Many elderly people find this difficult to accept and do not persevere enough, though it would undoubtedly help them to do some reading. Some of the new low-vision aids include a screen, like a television screen, on which reading matter is shown in magnified form, and this does give a bigger field, but unfortunately they are expensive. It is certain, however, that much can be done to help registered blind persons to read, whether by the use of Braille or by other means, and everyone with defective reading vision should be given the opportunity of employing one of these methods.

\section{Extracorporeal Oxygenation for Acute Respiratory Failure}

The commonest torm of acute respiratory failure in Britain is that associated with chronic airways obstruction; characteristically there is a fall in arterial $\mathrm{PO}_{2}$ and a rise in arterial $\mathrm{PCO}_{2}$. Though arguments persist about the optimum level of arterial $\mathrm{PO}_{2}$ in such patients, it is not difficult to improve arterial oxygenation, often with relatively modest increases in inspired oxygen concentration. ${ }^{1}$ The major therapeutic problem is that as the inspired oxygen is raised there may be an accompanying rise in arterial $\mathrm{PCO}_{2}$ and increasing drowsiness. For such patients mechanical ventilation may be necessary; this will almost always correct carbon dioxide retention-indeed care has to be taken to avoid too drastic a reduction ${ }^{2}$ in arterial $\mathrm{PCO}_{2}$.

But there is another type of respiratory failure seen in acutely ill patients, often without preceding lung disease, in which there is extremely severe hypoxaemia without $\mathrm{CO}_{2}$ retention. Its causes include severe pneumonia, trauma to the lung, fat or amniotic fluid embolism, and "shock lung" associated with severe non-thoracic trauma or septicaemia. The first step in such patients is to increase the inspired oxygen concentration. If an adequate level of arterial $\mathrm{Po}_{2}$ (which should be higher in these patients than in patients adapted to chronic hypoxia) cannot be achieved with an inspired oxygen concentration of $50-60 \%$, this implies that much of the pulmonary blood flow is passing through the lung without coming into contact with alveolar gas. Further increases in inspired oxygen concentration are unlikely to produce much improvement in arterial $\mathrm{Po}_{2}$ and carry the potential hazard of increasing the lung injury. ${ }^{3}$ In this situation mechanical ventilation with positive end-expiratory pressure usually improves arterial $\mathrm{Po}_{2}$ and may also allow a lower concentration of inspired oxygen. ${ }^{4}$

In a few patients these measures fail to achieve adequate arterial oxygenation; in this critical situation prolonged extracorporeal support with a membrane oxygenator has been proposed. Most experience with this technique has been acquired in the United States, and a recent review reported 15 long-term survivors out of 130 patients submitted to the procedure. ${ }^{5}$ This survival rate is encouraging-the selection criteria were sufficiently severe to ensure that only patients with extremely widespread and intractable lung disorders were treated. The technical problem of maintaining adequate oxygenation for several days seems to be well on the way to solution, but heparinization is required and problems persist with haemorrhage, thrombocytopenia, and infection. As in the early days of other organ support systems a major problem has been to define which overwhelming (and usually rapidly fatal) lung conditions are reversible with time and which are not. So far the best results have been obtained with "shock lung" after trauma or with pulmonary fat emboli. ${ }^{6}$ Disappointingly, the results with severe bacterial and viral pneumonias have been poor. ${ }^{78}$

It is apparent that a prodigious research effort will be required to establish extracorporeal oxygenation as a practical procedure and to clarify the indications for its use. Probably only a few centres should attempt to acquire the necessary practical experience in the technique; in the United States the National Heart and Lung Institute is supporting research programmes in nine units. Inevitably the procedure will remain costly, but patients with the type of respiratory failure likely to benefit from this treatment are often young and without pre-existing disease. Moreover the technique would be an invaluable adjunct if lung transplantation became a definitive treatment for irreversible respiratory failure.

${ }^{1}$ Campbell, E. J. M., American Review of Respiratory Diseases, 1967, 96, 626. 2 Sykes, M. K., McNicol, M. W., and Campbell, E. J. M., Respiratory Failure. Oxford, Blackwell Scientific Publications, 1968, pp. 128 and 262.

3 Winter, P. M., and Smith, G., Anesthesiology, 1972, 37, 210.

4 Ashbaugh, D. G., et al., Lancet, 1967, 2, 319.

5 Hill, J. D., et al., fournal of Thoracic and Cardiovascular Surgery, 1974, 68, 905.

${ }^{6}$ Hill, J. D., et al., New England Fournal of Medicine, 1972, 286, 629.

7 Lefrak, E. A., et al., Chest, 1974, 66, 385.

8 Bartlett, R. H., et al., Fournal of Thoracic and Cardiovascular Surgery, $1974,68,918$. 